\title{
Additive effects of postchallenge hyperglycemia and low-density lipoprotein particles on the risk of arterial stiffness in healthy adults
}

\author{
Cheng Ding ${ }^{1}$, Sandy Huey-Jen $\mathrm{Hsu}^{2}$, Yong-Jian $\mathrm{Wu}^{{ }^{*}}$ and Ta-Chen Su${ }^{3 *}$
}

\begin{abstract}
Background: To determine the effects of post-challenge hyperglycemia potentiate low-density lipoprotein cholesterol $(L D L)$ particles on the risk of arterial stiffness in non-diabetic adults.

Methods: During 2009-2011, 592 adults without clinical diabetes (fasting glucose $<7.0$ mmol/L) or known coronary heart disease or stroke were recruited. All subjects underwent standard 75-g oral glucose tolerance test (OGTT) after overnight fasting. The glucose area under curve (GluAUC) after OGTT was defined as the postchallenge glucose load. Levels of LDL-C and small dense LDL-C (sdLDL-C) were measured. Arterial stiffness in terms of brachial-ankle pulse wave velocity (baPW) was also measured.

Results: The baPWV in tertile distributions were significantly associated with all conventional cardiovascular risk factors, $\mathrm{LDL}-\mathrm{C}$, and sdLDL-C. Multivariate logistic regression analyses revealed that $\mathrm{LDL}-\mathrm{C}$ (or sdLDL-C) combined with one of the seven glycemic indices (glucose levels at 0, 30, 60, 90, and 120 min; GluAUC; HbA1C) was associated with arterial stiffness after covariates being adjusted. Further interaction analyses showed only concurrent higher levels of both glycemic indices and atherogenic LDL-C or sdLDL-C have significant risk for arterial stiffness.

Conclusions: Additive effects of both postchallenge hyperglycemia and LDL subclass particles potentiate the risk of arterial stiffness. The adverse joint effects of hyperlipidemia and postchallenge hyperglycemia on subclinical cardiovascular function provide important information in primary prevention of cardiovascular disease in subjects without clinical diabetes.
\end{abstract}

Keywords: Postchallenge hyperglycemia, sdLDL-C, LDL-C, Arterial stiffness, OGTT

\section{Background}

Several epidemiological and pathological studies have shown that diabetes is an independent risk factor for cardiovascular disease (CVD) in men and women [1]. As the recommendation from the 2002 National Cholesterol Education Program Adult Treatment Panel III, diabetes mellitus has been considered as a coronary heart disease (CHD) equivalent [2], although residual controversy has continued into this decade. Recently, it was demonstrated

\footnotetext{
* Correspondence: fuwaihospital@gmail.com; tachensu@ntu.edu.tw ${ }^{1}$ State Key Laboratory of Cardiovascular Disease, Department of Cardiology, Cardiovascular Institute, Fuwai Hospital and National Center for Cardiovascular Diseases, Chinese Academy of Medical Sciences \& Peking Union Medical College, Beijing 100037, China

${ }^{3}$ Departments of Internal Medicine and Cardiovascular Center, National Taiwan University Hospital, 7, Chung-Shan South Road, Taipei 100 10002, Taiwan

Full list of author information is available at the end of the article
}

that some diabetic $\mathrm{CHD}$ patients may be at a greater risk of CVD morbidity and mortality compared with nondiabetic CHD patients [3]. Moreover, the Heart Protection Study provides direct evidence that cholesterol-lowering therapy is beneficial for people with diabetes even if they have not developed CHD or display elevated cholesterol concentrations [4]. Another study also showed that cholesterol-lowering therapy is efficacious in reducing the risk of first CVD events in patients with type 2 diabetes without high low-density lipoprotein (LDL) cholesterol (LDL-C) levels [5]; however, the interaction between glucose and the cholesterol concentrations remains unclear. On the other hand, about one-third of American adults and two-thirds of CHD patients have abnormal glu- 
cose metabolism [6,7]. Most of these high-risk individuals are still within nondiabetic glucose levels (fasting plasma glucose, FPG $<7.0 \mathrm{mmol} / \mathrm{l}$ or 2 -h plasma glucose $<11.1 \mathrm{mmol} / \mathrm{l})$, but show prediabetes classification with impaired glucose tolerance (FPG $<7.0 \mathrm{mmol} / \mathrm{l}$ and 2-h plasma glucose $\geq 7.8$ and $<11.1 \mathrm{mmol} / \mathrm{l}$ ) or diabetes classification after an oral glucose tolerance test (2-h plasma glucose $>11.1 \mathrm{mmol} / \mathrm{l}$ ) or an ingestion of a meal. Several studies have shown that postchallenge glucose levels are more strongly associated with CVD compared with fasting glucose levels $[8,9]$, besides, glucose levels after a glucose challenge exhibit continuous linear relationships with both CVD death and all-cause mortality risk [10]. Alternatively, the importance of 2-h plasma glucose levels during oral glucose tolerance test (OGTT) is well established, but the importance of postchallenge hyperglycemia remains unclear [11].

Although the above studies indicated the importance of postprandial glucose levels, fasting plasma glucose and glycated hemoglobin (HbA1c) levels still play important roles in the assessment of glycemic levels, whereas postchallenge glucose values are often neglected [12]. This may be because of the inconvenience and cost of measuring these parameters in daily clinical practice [13]. The most common clinical phenotypes of dyslipidemia in individuals with impaired glucose metabolism include low high-density lipoprotein cholesterol (HDL-C) levels, hypertriglyceridemia, and a higher ratio of small dense LDL-cholesterol (sdLDL-C) [14-17]. Higher levels of sdLDL particles are considered to be more atherogenic compared with large buoyant LDL-C particles; therefore, sdLDL-C is considered to be an important and independent predictor of CVD $[18,19]$.

Brachial-ankle pulse wave velocity (baPWV) can be noninvasively measured simply by wrapping the four extremities with blood pressure cuffs, and it serves as a simple marker of vascular damage and cardiovascular risk [20,21]. Earlier studies on experimental atherosclerosis in monkeys demonstrated that the aortic pulse wave velocity (PWV) increased with the development of atherosclerosis [22]. The Rotterdam study, a population-based study including more than 3,000 subjects, demonstrated a strong association of aortic PWV with intima-media thickness (IMT) and the severity of plaques as measured by ultrasonographic tests [23]. In addition, from our previously work, which have demonstrated that postchallenge hyperglycemic spikes correlate with arterial stiffness which was independent of confounding factors in adults without diabetes [24].

Thus, we propose that measuring sdLDL particle concentrations in a cohort study involving individuals without clinical diabetes would be helpful in evaluating its earlier role of dyslipidemia-glucose hypothesis in atherosclerosis; we conducted this study among healthy adults without diabetes with the aim of investigating whether postchallenge hyperglycemia and LDL subclass particles exerted additive effects on arterial stiffness.

\section{Results}

The cardiovascular characteristics of all participants were compared according to the tertile distribution of sdLDL levels; subjects without previously known clinical diabetes that displayed 2-h glucose $\geq 11.1 \mathrm{mmol} / \mathrm{l}$ after OGTT were considered OGTT diabetes (Table 1). A strong correlation between different glycemic indices such as OGTT diabetes, GluAUC and fasting glucose across sdLDL-C tertile (Table 1) was noted. The conventional risk factors for CVD and diabetes, except smoking and alcohol consumption, showed statistically significant differences in the trend test across the three groups. Subjects with higher sdLDL levels exhibited more conventional risk factor for CVD and diabetes. Higher LDL-C and sdLDL/LDL ratios were also noted across the higher sdLDL levels. Most conventional risk factors and the novel risk factors of sdLDL and GluAUC were significantly associated with baPWV across the tertile distribution (Table 2). The upper tertile of baPWV subjects associated with significantly higher glucose profiles including OGTT diabetes, FPG, GluAUC and $\mathrm{HbA} 1 \mathrm{C}$ and lipid profiles such as cholesterol, triglyceride, LDL-C, sdLDL-C and sdLDL-C/LDL-C ratio. In contrast, levels of large LDL-C were borderline but significantly associated with baPWV. Additionally, there are no significant associations between baPWV and statin-users, and between baPWV and renal function index of estimated glomerular filtration rate.

Multivariate logistic regression models were applied to elucidate the impact of different glycemic indices, including post-OGTT glucose levels, GluAUC, and HbA1C, combined with LDL-C (Table 3) or with sdLDL-C (Table 4) on the risk of baPWV. The results demonstrated that fasting glucose levels were not associated with arterial stiffness after controlling for other covariates; however, postchallenge glucose levels at 30,60, 90, and $120 \mathrm{~min}$ and GluAUC were significantly and positively associated with arterial stiffness. Furthermore, the joint effects of postchallenge glucose levels and GluAUC with either LDL-C or sdLDL-C were significantly linked to arterial stiffness. On the other hand, when the joint effects of both LDL-C and fasting glucose levels or LDL-C and HbA1C were considered, the two glycemic indices no longer had a significant influence on increased baPWV (Table 3). Similar findings were observed when LDL-C was replaced with sdLDL-C in the same multivariate models (Table 4). Fasting glucose and $\mathrm{HbA1C}$ did not significantly influence the risk of arterial stiffness in the initial multivariate analyses (Tables 3 and 4).

However, further multivariate analyses using interaction analyses between glycemic indices and atherogenic 
Table 1 Cardiovascular characteristics of participants according to tertile distribution of small dense-low-density lipoprotein cholesterol (sdLDL-C)

\begin{tabular}{|c|c|c|c|c|}
\hline \multirow[b]{4}{*}{ Characteristics } & \multicolumn{3}{|c|}{ sdLDL-C, mmol/L } & \multirow{4}{*}{$p$-value } \\
\hline & Upper & Middle & Lower & \\
\hline & $\geq 37.7$ & $24.0-37.7$ & $<24.0$ & \\
\hline & $N=197$ & $\mathrm{~N}=197$ & $N=198$ & \\
\hline Age, years & $45.82 \pm 7.61$ & $44.41 \pm 7.96$ & $45.13 \pm 7.99$ & 0.390 \\
\hline Male, \% & 91.88 & 90.16 & 78.35 & $<.001$ \\
\hline $\mathrm{BMI}, \mathrm{kg} / \mathrm{m}^{2}$ & $25.79 \pm 2.95$ & $25.26 \pm 3.62$ & $24.19 \pm 3.19$ & $<.001$ \\
\hline Waist, $\mathrm{cm}$ & $87.28 \pm 7.94$ & $85.61 \pm 10.1$ & $81.87 \pm 9.04$ & $<.001$ \\
\hline Smoking, \% & 20.81 & 14.95 & 14.29 & 0.083 \\
\hline Alcohol, \% & 23.86 & 21.65 & 14.8 & 0.025 \\
\hline Hypertension, \% & 27.92 & 18.04 & 14.8 & 0.001 \\
\hline Systolic BP, mmHg & $126.04 \pm 14.67$ & $121.23 \pm 10.91$ & $117.44 \pm 13.23$ & $<.001$ \\
\hline Diastolic BP, mmHg & $77.53 \pm 9.79$ & $74.18 \pm 8.19$ & $71.28 \pm 9.51$ & $<.001$ \\
\hline OGTT diabetes, \% & 9.14 & 4.12 & 3.57 & 0.017 \\
\hline Fasting glucose, mmol/L & $5.45 \pm 1.21$ & $5.15 \pm 0.78$ & $5.03 \pm 0.93$ & $<.001$ \\
\hline GluAUC, mmol/L & $32.53 \pm 10.07$ & $29.24 \pm 7.01$ & $26.64 \pm 8.13$ & $<.001$ \\
\hline $\mathrm{HbA} 1 \mathrm{C}, \%$ & $5.85 \pm 1.05$ & $5.55 \pm 0.46$ & $5.56 \pm 0.69$ & $<.001$ \\
\hline Cholesterol, mmol/L & $5.98 \pm 0.88$ & $5.31 \pm 0.62$ & $4.7 \pm 0.65$ & $<.001$ \\
\hline Triglyceride, mmol/L & $2.39 \pm 1.49$ & $1.48 \pm 0.75$ & $0.99 \pm 0.41$ & $<.001$ \\
\hline $\mathrm{HDL}-\mathrm{C}, \mathrm{mmol} / \mathrm{L}$ & $1.22 \pm 0.3$ & $1.27 \pm 0.31$ & $1.37 \pm 0.3$ & $<.001$ \\
\hline LDL-C, mmol/L & $3.55 \pm 0.89$ & $3.15 \pm 0.61$ & $2.59 \pm 0.52$ & $<.001$ \\
\hline sdLDL-C/LDL-C & $0.39 \pm 0.13$ & $0.26 \pm 0.07$ & $0.18 \pm 0.04$ & $<.001$ \\
\hline
\end{tabular}

OGTT Diabetes: subjects' results with OGTT two hour value $>11.1 \mathrm{mmol} / \mathrm{l}$.

Abbreviations: OGTT oral glucose tolerance test, GluAUC glucose area under curve after OGT.

lipoprotein particles revealed that that only group of concurrent with highest tertile levels of LDL-C (or sdLDL-C) and higher half levels glycemic indices have significant risk for arterial stiffness and this is independent of fasting status (either fasting or postchallenge) or HbA1C levels (Table 5).

\section{Discussion}

To the best of our knowledge, the present study is the first to demonstrate the concurrent presence of both atherogenic lipoprotein particles (LDL and sdLDL) and postchallenge hyperglycemia as risk factors of arterial stiffness in nondiabetic adults. The novel finding of significant interactions between LDL subclass particles and different glycemic indices also indicate an additive effect between these two risk factors on arterial stiffness. In addition to postchallenge glucose levels, both LDL-C and sdLDL-C levels were significantly related to an increased risk of arterial stiffness. Another novel result of our study found that sdLDL-C might play an important role as LDL-C as it being closely related to the early atherosclerosis maker of baPWV.

Formerly studies have demonstrated that postchallenge hyperglycemia status predisposes a higher risk of atherosclerosis or CVD that can be attributed to the rapid increase in oxidative stress after meals or post glucose load [25]. Therefore, postchallenge hyperglycemia may trigger the interaction between oxidative stress, remnant-like lipoproteins, LDL-C, and sdLDL and subsequently elevate the risk of cardiovascular complications. Our study showed that postchallenge hyperglycemia at all the different time-course (30,60, 90, and $120 \mathrm{~min})$ and GluAUC was strongly related to arterial stiffness; therefore, it is reasonable to postulated that postchallenge hyperglycemia could have additive effects with atherogenic lipoprotein particles (LDL and sdLDL) on subclinical atherosclerosis, former research have indicated that glucosemediated elastic artery sclerosis may play an integral role in the development of macrovascular complications [26]. The additive effects of atherogenic LDL-C (or sdLDL-C) and different glycemic indices on arterial stiffness demonstrated that postchallenge hyperglycemia have similar effects on subclinical atherogenesis compared with fasting glucose or HbA1C, particularly in subjects with higher sdLDL-C or LDL-C levels.

Additionally, our study also provides direct evidence that the occurrence of hyperglycemia, which was independent from the status of postchallenge, fasting, or 
Table 2 Cardiovascular characteristics of participants according to tertile distribution of brachial-ankle pulse wave velocity (baPWV)

\begin{tabular}{|c|c|c|c|c|}
\hline \multirow[b]{4}{*}{ Characteristics } & \multicolumn{3}{|c|}{ baPWV } & \multirow{4}{*}{$p$-value } \\
\hline & Upper & Middle & Lower & \\
\hline & $\geq 1438.3$ & $1322.5-1438.3$ & $<1322.5$ & \\
\hline & $\mathrm{N}=198$ & $N=197$ & $N=197$ & \\
\hline Age, years & $48.18 \pm 7.35$ & $45.12 \pm 7.53$ & $42.24 \pm 7.58$ & $<.001$ \\
\hline Male, \% & 92.93 & 87.24 & 81.03 & 0.004 \\
\hline $\mathrm{BMI}, \mathrm{kg} / \mathrm{m}^{2}$ & $25.43 \pm 3.23$ & $25.27 \pm 3.26$ & $24.58 \pm 3.48$ & 0.010 \\
\hline Waist, cm & $86.34 \pm 8.48$ & $85.23 \pm 9.06$ & $83.37 \pm 10.17$ & 0.001 \\
\hline Smoking, \% & 20.2 & 13.71 & 16.75 & 0.359 \\
\hline Alcohol, \% & 22.22 & 21.83 & 15.23 & 0.081 \\
\hline Hypertension, \% & 33.84 & 17.77 & 8.12 & $<.001$ \\
\hline Systolic BP, mmHg & $129.61 \pm 14.58$ & $121.52 \pm 10.71$ & $113.59 \pm 9.25$ & $<.001$ \\
\hline Diastolic BP, mmHg & $79.50 \pm 9.71$ & $75.02 \pm 7.85$ & $68.59 \pm 7.44$ & $<.001$ \\
\hline OGTT diabetes, \% & 9.6 & 4.06 & 1.52 & $<.001$ \\
\hline Fasting glucose, $\mathrm{mmol} / \mathrm{L}$ & $5.39 \pm 0.92$ & $5.18 \pm 0.89$ & $4.99 \pm 0.86$ & $<.001$ \\
\hline GluAUC, mmol/L & $32.38 \pm 9.84$ & $29.09 \pm 7.82$ & $27.37 \pm 7.97$ & $<.001$ \\
\hline $\mathrm{HbA} 1 \mathrm{C}, \%$ & $5.77 \pm 0.87$ & $5.62 \pm 0.57$ & $5.51 \pm 0.72$ & $<.001$ \\
\hline Cholesterol, mmol/L & $5.50 \pm 0.96$ & $5.37 \pm 0.93$ & $5.14 \pm 0.74$ & $<.001$ \\
\hline Triglyceride, mmol/L & $1.85 \pm 1.43$ & $1.61 \pm 0.94$ & $1.41 \pm 0.97$ & $<.001$ \\
\hline $\mathrm{HDL}-\mathrm{C}, \mathrm{mmol} / \mathrm{L}$ & $1.24 \pm 0.27$ & $1.30 \pm 0.32$ & $1.33 \pm 0.33$ & 0.005 \\
\hline LDL-C, mmol/L & $3.28 \pm 0.87$ & $3.12 \pm 0.76$ & $2.90 \pm 0.71$ & $<.001$ \\
\hline sdLDL, mmol/L & $0.96 \pm 0.47$ & $0.86 \pm 0.41$ & $0.73 \pm 0.33$ & $<.001$ \\
\hline Large LDL-C, mmol/L & $2.31 \pm 0.71$ & $2.28 \pm 0.64$ & $2.18 \pm 0.60$ & 0.037 \\
\hline sdLDL-C/LDL-C & $0.30 \pm 0.14$ & $0.27 \pm 0.12$ & $0.25 \pm 0.11$ & $<.001$ \\
\hline $\mathrm{eGFR}, \mathrm{ml} / \mathrm{min}$ & $87.26 \pm 19.60$ & $88.91 \pm 20.49$ & $89.39 \pm 20.05$ & 0.323 \\
\hline Statin-users, \% & 13.13 & 8.63 & 9.64 & 0.257 \\
\hline
\end{tabular}

OGTT Diabetes: subjects' results with OGTT two hour value $>11.1 \mathrm{mmol} / \mathrm{l}$.

Abbreviations: OGTT oral glucose tolerance test, GluAUC glucose area under curve after OGTT, sdLDL-C small dense LDL-C, Large LDL-C $=L D L-C-s d L D L-C$, eGFR estimated glomerular filtration rate.

Table 3 Multiple logistic regression analyses for the risk [odds ratios (95\% confidence intervals)] of higher brachial-ankle pulse wave velocity (baPWV $\geq 75^{\text {th }}$ percentile) focused on the combined effects of LDL-C and different glycemic indices

\begin{tabular}{|c|c|c|c|c|c|c|c|c|}
\hline & Basic models ${ }^{a}$ & Model 1 & Model 2 & Model 3 & Model 4 & Model 5 & Model 6 & Model 7 \\
\hline LDL-C & $1.62(1.27-2.06)^{\ddagger}$ & $1.59(1.24-2.02)^{\ddagger}$ & $1.55(1.21-1.99)^{\ddagger}$ & $1.53(1.19-1.95)^{\ddagger}$ & $1.56(1.22-1.99)^{\ddagger}$ & $1.57(1.23-2.00)^{\ddagger}$ & $1.54(1.20-1.97)^{\ddagger}$ & $1.60(1.25-2.04)^{\ddagger}$ \\
\hline \multicolumn{9}{|l|}{ Glucose, } \\
\hline $0 \min$ & $1.24(0.99-1.55)^{*}$ & $1.19(0.96-1.48)$ & - & - & - & - & - & - \\
\hline $30 \mathrm{~min}$ & $1.23(1.10-1.38)^{*}$ & - & $1.21(1.08-1.35)^{\dagger}$ & - & - & - & - & - \\
\hline $60 \mathrm{~min}$ & $1.15(1.07-1.23)^{*}$ & - & - & $1.13(1.05-1.22)^{*}$ & - & - & - & - \\
\hline $90 \mathrm{~min}$ & $1.12(1.05-1.20)^{*}$ & - & - & - & $1.10(1.03-1.18)^{*}$ & - & - & - \\
\hline $120 \mathrm{~min}$ & $1.12(1.04-1.20)^{*}$ & - & - & - & - & $1.10(1.02-1.19)^{*}$ & - & - \\
\hline GluAUC & $1.09(1.04-1.14)^{*}$ & - & - & - & - & - & $1.08(1.03-1.13)^{*}$ & - \\
\hline $\mathrm{HbA1C}$ & $1.34(1.02-1.76)^{*}$ & - & - & - & - & - & - & $1.30(0.99-1.72)$ \\
\hline
\end{tabular}

abasic models included LDL-C or one of the seven glycemic indices after controlling for age, male gender, BMl, hypertension, smoking, and alcohol consumption habit. p-value: ${ }^{*}<0.05,{ }^{\dagger}<0.01,{ }^{\ddagger}<0.001$.

Models 1 to 7 combined both LDL-C and one of the seven glycemic indices after controlling for age, male gender, BMI, hypertension, smoking, and alcohol consumption. 
Table 4 Multiple logistic regression analyses for the risk [odds ratios (95\% confidence intervals)] of higher brachial-ankle pulse wave velocity (baPWV $\geq 75^{\text {th }}$ percentile) focused on the combined effects of sdLDL-C and different glycemic indices

\begin{tabular}{|c|c|c|c|c|c|c|c|c|}
\hline & Basic models $^{a}$ & Model 1 & Model 2 & Model 3 & Model 4 & Model 5 & Model 6 & Model 7 \\
\hline sdLDL-C & $2.51(1.56-4.05)^{\ddagger}$ & $2.38(1.46-3.87)^{\ddagger}$ & $2.17(1.32-3.57)^{\ddagger}$ & $2.12(1.29-3.48)^{\ddagger}$ & $2.22(1.36-3.64)^{\ddagger}$ & $2.30(1.42-3.75)^{\ddagger}$ & $2.16(1.31-3.54)^{\ddagger}$ & $2.37(1.46-3.86)^{\ddagger}$ \\
\hline \multicolumn{9}{|l|}{ Glucose, } \\
\hline $0 \mathrm{~min}$ & $1.24(0.99-1.54)$ & $1.15(0.93-1.43)$ & - & - & - & - & - & - \\
\hline $30 \mathrm{~min}$ & $1.23(1.10-1.38)^{\dagger}$ & - & $1.18(1.05-1.33)^{\dagger}$ & - & - & - & - & - \\
\hline $60 \mathrm{~min}$ & $1.15(1.07-1.23)^{*}$ & - & - & $1.12(1.04-1.20)^{*}$ & - & - & - & - \\
\hline $90 \mathrm{~min}$ & $1.12(1.04-1.20)^{*}$ & - & - & - & $1.09(1.02-1.17)^{*}$ & - & - & - \\
\hline $120 \mathrm{~min}$ & $1.11(1.03-1.20)^{*}$ & - & - & - & - & $1.09(1.01-1.18)^{*}$ & - & - \\
\hline GluAUC & $1.09(1.04-1.14)^{*}$ & - & - & - & - & - & $1.07(1.02-1.12)^{*}$ & - \\
\hline $\mathrm{HbA} 1 \mathrm{C}$ & $1.36(1.02-1.75)^{*}$ & - & - & - & - & - & - & $1.22(0.93-1.60)$ \\
\hline
\end{tabular}

${ }^{\mathrm{a}}$ Basic models included sdLDL-C or one of the seven glycemic indices after controlling for age, male gender, BMI, hypertension, smoking, and alcohol consumption habit. p-value: ${ }^{*}<0.05,{ }^{\dagger}<0.01,{ }^{\ddagger}<0.001$.

Models 1 to 7 combined both sdLDL-C and one of the seven glycemic indices after controlling for age, male gender, BMl, hypertension, smoking, and alcohol consumption.

HbA1C, may potentiate the effect of atherogenic lipoprotein particles (LDL and sdLDL) on the risk of subclinical atherosclerosis. The findings may also further support the results of elegant large-scale lipid-lowering trials among diabetes that demonstrate that with statin treatments can significantly slowing atherogenic progress. Thus, may contribute the reduction of cardiovascular morbidity and mortality risk [27].

Moreover, our results also are in agreement with those previous studies reported that an increase in sdLDL particles and increased postchallenge hyperglycemia may lead to an elevated risk of subclinical CVD $[28,29]$. Also, based on LDL particle size has been found to be the strongest marker of clinically apparent and nonapparent atherosclerosis in patients with type 2 diabetes [30]. These studies further displayed that quantitative sdLDL$\mathrm{C}$ measurements could provide alternative useful information for the risk assessment of atherosclerotic disease in addition to LDL-C [31].
It is well known that diabetes is a complicated disease that involves different levels of cellular dysfunction and multiple organs; hyperglycemia accelerated arterial stiffening by increasing the formation of AGE, which alters vessel wall structure and function. The irreversible formation and deposition of reactive AGE may most likely be the pathway involved in the pathogenesis of accelerated atherosclerosis in patients with diabetes [32]. The atherogenic effects of both fasting and postprandial hyperglycemia were ascribed to glucose-mediated cellular properties via several mechanisms such as an increase in nonenzymatic glycation of proteins and lipids. The findings of this study enhanced our previous work that postchallenge hyperglycemia is associated with the subclinical atherosclerotic marker cardio-ankle vascular index of arterial stiffness [24]. In another study involving 582 individuals aged $40-70$ years and at risk for type 2 diabetes, postchallenge plasma glucose levels $(30,60,90$, and $120 \mathrm{~min}$ ) as well as GluAUC were more strongly

Table 5 Multivariate logistic regression analyses for the interaction between atherogenic lipids and glycemic indices (GI) on the risk of arterial stiffness (baPWV $\geq 75^{\text {th }}$ percentile)

\begin{tabular}{|c|c|c|c|c|c|c|c|}
\hline & $\mathrm{AC}$ & Glucose 30 & Glucose 60 & Glucose 90 & Glucose 120 & Glucose AUC & $\mathrm{HbA1C}$ \\
\hline LDL-C $\geq 2 / 3 \&$ Gl $\geq 50 \%$ & $2.63(1.59,4.35)^{\ddagger}$ & $2.13(1.29,3.52)^{\dagger}$ & $2.26(1.36,3.75)^{\dagger}$ & $1.85(1.12,3.06)^{\dagger}$ & $2.21(1.34,3.65)^{\dagger}$ & $2.27(1.38,3.74)^{\dagger}$ & $2.50(1.49,4.19)^{\ddagger}$ \\
\hline LDL-C $\geq 2 / 3 \& \mathrm{GI}<50 \%$ & $1.69(0.99,2.89)$ & $1.28(0.74,2.21)$ & $1.15(0.67,1.97)$ & $1.55(0.90,2.65)$ & $1.78(1.04,3.04)^{*}$ & $1.40(0.81,2.41)$ & $1.89(1.13,3.16)^{*}$ \\
\hline LDL-C $<2 / 3 \&$ GI $\geq 50 \%$ & $1.16(0.75,1.80)$ & $0.68(0.43,1.08)$ & $0.65(0.41,1.02)$ & $0.71(0.45,1.12)$ & $0.98(0.63,1.54)$ & $0.84(0.53,1.32)$ & $1.18(0.76,1.85)$ \\
\hline LDL-C $<2 / 3 \& \mathrm{GI}<50 \%$ & 1 & 1 & 1 & 1 & 1 & 1 & 1 \\
\hline sdLDL-C $\geq 2 / 3 \&$ Gl $\geq 50 \%$ & $2.05(1.26,3.33)^{\dagger}$ & $1.70(1.02,2.82)^{*}$ & $1.63(1.00,2.66)^{*}$ & $1.47(0.91,2.40)$ & $1.74(1.06,2.84)^{*}$ & $1.84(1.12,3.02)^{\dagger}$ & $1.97(1.18,3.29)^{\dagger}$ \\
\hline sdLDL-C $\geq 2 / 3 \&$ GI $<50 \%$ & $1.20(0.68,2.13)$ & $0.79(0.51,1.23)$ & $1.34(0.76,2.35)$ & $1.25(0.70,2.21)$ & $1.38(0.79,2.41)$ & $1.10(0.62,1.94)$ & $1.50(0.89,2.54)$ \\
\hline sdLDL-C $<2 / 3 \& \mathrm{GI} \geq 50 \%$ & $1.12(0.72,1.73)$ & $1.11(0.64,1.93)$ & $0.87(0.56,1.36)$ & $0.71(0.46,1.12)$ & $0.99(0.64,1.54)$ & $0.88(0.56,1.38)$ & $1.21(0.78,1.88)$ \\
\hline sdLDL-C $<2 / 3 \& \mathrm{Gl}<50 \%$ & 1 & 1 & 1 & 1 & 1 & 1 & 1 \\
\hline
\end{tabular}

p-value: ${ }^{*}<0.05,{ }^{\dagger}<0.01,{ }^{\ddagger}<0.001$

All models were after adjusting for age, male gender, BMl, hypertension, alcohol drinking and current smoker, in addition to glycemic indices and LDL-C (or sdLDL-C). Levels of LDL-C (130 mg/dL) and sdLDL-C ( $37 \mathrm{mg} / \mathrm{dL})$ were set at cut point of $\geq$ upper tertile (2/3) $\left(66.7^{\text {th }}\right.$ percentile) in the models. 
associated with carotid IMT compared with fasting glucose and HbA1c levels [28]. However, applying interaction analyses to test the concurrent atherogenic lipids and glycemic indices provides a deeper view of the interaction between lipids and hyperglycemia on the development of atherosclerosis.

Studies from other Asian populations have demonstrated that postchallenge glucose levels may be related to CVD events in patients without diabetes [33,34]. Combining the additive effects atherogenesis observed during the hyperglycemic state of the present study also showed a higher prevalence of cardiovascular risk factors among patients belonging to the highest tertile. It is reasonable to assume that the postchallenge hyperglycemic spikes may account for the aforementioned increased CVD events. Therefore, postchallenge hyperglycemia should be emphasized as a target for decreasing the incidence of diabetes and CVD [35].

Recently, a research conducted in a type 2 diabetes and prediabetes population has successfully displayed the relationship between sdLDL with carotid IMT and insulin resistance [36]. In addition to the traditional risk markers, sdLDL has become an independent risk marker for adverse metabolic status such as dysglycemia, indicating that more attention should be paid to hyperglycemia which occurs independently from fasting, postchallenge, or postprandial in subjects with atherogenic dyslipidemia and particularly high levels of LDL-C or sdLDL-C to serve as a primary prevention of CVD.

The present study should be interpreted in consideration of some limitations. First, the study conducted within non-diabetes patients instead of diabetes patients. However, in order to eliminate confounding factors such as chronic diabetes-related complications and interventions, our study evaluated information gathered from adults without diabetes. Second, even though HbA1c was not statistically and significantly associated with baPWV in the presented study, based on this crosssectional study, we cannot definitely make the conclusion that postprandial hyperglycemia are more important than $\mathrm{HbA1C}$ in the association with cardiovascular diseases. Last, the exact mechanisms of interactions between glucose and lipids profiles need to be elucidated in future study.

\section{Conclusions}

In conclusion, significant interactions between LDL subclass particles and different glycemic indices displayed an additive effect between these two risk factors on arterial stiffness. In addition, only concurrent highest tertile levels of both glycemic indices and atherogenic LDL-C or sdLDL-C have significant risk for arterial stiffness in nondiabetic adults.

\section{Methods}

From 2009 to 2011, we recruited 592 middle-aged (age range, 20-60 years old) adults without clinical diabetes (fasting plasma glucose $<7.0 \mathrm{mmol} / \mathrm{L}$ or 2 -h plasma glucose $<11.1 \mathrm{mmol} / \mathrm{L}$ after OGTT) or known history of CHD or stroke to participate as the control group of "Work-related factors and cardiovascular events in patients with coronary heart disease" study conducted in the National Taiwan University Hospital, Taipei, Taiwan [37]. The present study of below diagnostic OGTT diabetes criteria was defined as 2 -h glucose $\geq 11.1 \mathrm{mmol} / \mathrm{L}$ after OGTT but without previously known clinical diabetes as above mentioned. Informed consent was obtained prior to participation. The body mass index was calculated by the formula: weight $(\mathrm{kg}) /[\text { height }(\mathrm{m})]^{2}$. Smoking habit was defined as persons who had smoked more than 100 cigarettes in their lifetimes and still smoke at the time of the study. Hypertension is defined as SBP values $>140 \mathrm{mmHg}$ and/or DBP $>90 \mathrm{mmHg}$, alcohol consumption. The alcohol consumption was defined as up to 1 drink per day for women and up to 2 drinks per day for men. This study was approved by the ethics committee of National Taiwan University Hospital.

\section{Blood sampling for lipids and biochemical studies}

Following overnight fasting for 10-14 h, the subjects underwent a standard 75-g oral OGTT to evaluate their response to glucose loading. A venous blood sample was obtained through the antecubital vein before OGTT and at 30, 60, 90, and $120 \mathrm{~min}$ after OGTT. The glucose area under curve (GluAUC) for each subject was calculated by the five times $(0,30,60,90$, and 120$)$ of the blood glucose level sample after OGTT. The postprandial period was defined to extend until $120 \mathrm{~min}$ after meal onset, baseline (0) was defined as the pre-meal plasma value. The assessment of levels of lipids, including cholesterol, triglycerides, HDL-C, and LDL-C, Cholesterol within small dense LDL $(15.0 \mathrm{~nm}-20.0 \mathrm{~nm})$ was measured as described previously $[38,39]$ using a newly developed automated homogeneous assay (Denka Seiken Co., Ltd., 3-4-2 Nihonbashi-Kayabacho, Chuo-Ku, Tokyo) and analyzed on an autoanalyzer (Toshiba FR-200 automatic chemistry analyzer, Tokyo, Japan). Subject's blood samples were collected in the morning under 10-14 $\mathrm{h}$ fasting conditions, after which the blood sample were centrifuged for $10 \mathrm{~min}$ at 3,000 revolutions per minute. The serum was separated and stored at $-80^{\circ} \mathrm{C}$. The blood collection to separation process did not exceed 3 hours under proper laboratory protocol.

\section{Brachial-ankle pulse wave velocity measurements}

The brachial-ankle pulse wave velocity (baPWV) was measured in the morning after participants received OGTT using a noninvasive vascular screening device 
(Colin VP-1000; Colin Co., Ltd., Komaki, Japan). This device simultaneously recorded the baPWV, electrocardiogram, and arterial blood pressure from all four limbs (at both the left and right brachia and ankles) and calculated the ankle-brachial index. Subjects were examined in the supine position. Electrodes were placed on both wrists to obtain an electrocardiogram. The cuffs were connected to a sensor that measured the volume pulse form and an oscillometric pressure sensor that measured blood pressure. The time interval between the wave front of the brachial waveform and the ankle waveform was defined as the pulse transit time between the brachia and the ankle $(\Delta \mathrm{Tba})$. The distance between the arm and ankle was estimated automatically according to the subject's height. The distance from the heart to the brachia $(\mathrm{Db})$ was measured using the following equation: $\mathrm{Db}=0.2195 \times$ height of the patient $(\mathrm{cm})-2.0734$. The distance from the heart to the ankle $(\mathrm{Da})$ was measured using the following equation: $\mathrm{Da}=0.8129 \times$ height of the patient $(\mathrm{cm})+12.328$. The baPWV was calculated using the following equation: baPWV $=(\mathrm{Da}-\mathrm{Db}) / \Delta \mathrm{Tb}$. The validity and reproducibility of baPWV measurements has been reported elsewhere [20,21].

\section{Statistical analysis}

Data analysis was performed using Statistical Analysis System (SAS) software (version 9.2, SAS Institute Inc, Cary NC, USA). The statistical significance level was set at 0.05 , and the power calculation was set with a 1-beta value of $80 \%$ for this study. Continuous variables were expressed as means \pm standard deviations and categorical data was expressed by percentage. The statistical methods used to test the significance of the compare continuous variables (Tables 1 and 2) were performed with $\mathrm{CA}$ (Cochran-Armitage) test for trend.

Basic information of cardiovascular characteristics and subclinical atherosclerotic markers was compared by Chi-square test for trend across the tertile distribution of the groups using glucose levels during postchallenge hyperglycemic spikes $1 \mathrm{~h}$ after the OGTT, which was performed to demonstrate postchallenge hyperglycemic status. The trend differences between the cardiovascular characteristics of the patients across the tertile distributions of the groups for baPWV were also evaluated.

The effects of LDL-C (or sdLDL-C) and glucose levels at the different time points of OGTT results were measured using multiple logistic regression models to estimate the odds ratios and 95\% confidence intervals for arterial stiffness in terms of baPWV at the level of $\geq 75$ th percentile. This analysis was performed after controlling for related covariates, including age, gender, BMI, hypertension, smoking, and alcohol consumption. Since there were high correlations observed between the different glycemic indices and atherogenic LDL-C (or sdLDL-C), the concurrent presence of these two components was treated as interaction to test their risk for arterial stiffness.

\section{Abbreviations}

CVD: Cardiovascular disease; CHD: Coronary heart disease; LDL: Low-density lipoprotein; LDL-C: Low-density lipoprotein cholesterol; FPG: Fasting plasma glucose; OGTT: Oral glucose tolerance test; HbA1c: Glycated hemoglobin; GluAUC: Glucose area under curve; HDL-C: High-density lipoprotein cholesterol; sdLDL-C: Small dense LDL-cholesterol; IMT: Intima-media thickness; baPWV: Brachial-ankle pulse wave velocity; eGFR: Estimated glomerular filtration rate.

\section{Competing interests}

The authors declare that they have no competing interests.

\section{Authors' contributions}

CD joined the research, drafted the manuscript. YJW reviewed/edited the manuscript. SHJH performed the laboratory examinations, including biochemical data and sdLDL-C. TCS designed and conducted the research, data analysis and interpretation, detailed discussion and drafted the manuscript, and reviewed/edited the manuscript. All authors read and approved the final manuscript. All authors read and approved the final manuscript.

\section{Acknowledgements}

This work was supported in part by the 3rd core facility at National Taiwan University Hospital. The National Science Council (NSC 101-2314-B-002-184-NY3) and National health Research Institute (NHRI-EX97-9721PC), Taiwan, and National Taiwan University (NTU-CESRP-102R7620) supported the study.

\section{Author details}

${ }^{1}$ State Key Laboratory of Cardiovascular Disease, Department of Cardiology, Cardiovascular Institute, Fuwai Hospital and National Center for

Cardiovascular Diseases, Chinese Academy of Medical Sciences \& Peking Union Medical College, Beijing 100037, China. ${ }^{2}$ Department of Laboratory Medicines, National Taiwan University Hospital, Taipei, Taiwan. ${ }^{3}$ Departments of Internal Medicine and Cardiovascular Center, National Taiwan University Hospital, 7, Chung-Shan South Road, Taipei 100 10002, Taiwan.

Received: 5 December 2013 Accepted: 21 November 2014 Published: 27 November 2014

\section{References}

1. Wilson PW, D'Agostino RB, Levy D, Belanger AM, Silbershatz H, Kannel WB: Prediction of coronary heart disease using risk factor categories. Circulation 1998, 97:1837-1847.

2. National Cholesterol Education Program (NCEP) Expert Panel on Detection, Evaluation, and Treatment of High Blood Cholesterol in Adults (Adult Treatment Panel III): Third report of the National Cholesterol Education Program (NCEP) expert panel on detection, evaluation, and treatment of high blood cholesterol in adults (adult treatment panel III) final report. Circulation 2002, 106:3143-3421.

3. Malmberg K, Yusuf S, Gerstein HC, Brown J, Zhao F, Hunt D, Piegas L, Calvin J, Keltai M, Budaj A: Impact of diabetes on long-term prognosis in patients with unstable angina and non-Q-wave myocardial infarction: results of the OASIS (Organization to Assess Strategies for Ischemic Syndromes) Registry. Circulation 2000, 102:1014-1019.

4. Collins R, Armitage J, Parish S, Sleigh P, Peto R, Heart Protection Study Collaborative G: MRC/BHF heart protection study of cholesterol-lowering with simvastatin in 5963 people with diabetes: a randomised placebocontrolled trial. Lancet 2003, 361:2005-2016.

5. Colhoun HM, Betteridge DJ, Durrington PN, Hitman GA, Neil HA, Livingstone SJ, Thomason MJ, Mackness MI, Charlton-Menys V, Fuller JH, investigators C: Primary prevention of cardiovascular disease with atorvastatin in type 2 diabetes in the Collaborative Atorvastatin Diabetes Study (CARDS): multicentre randomised placebo-controlled trial. Lancet 2004, 364:685-696.

6. Cowie CC, Rust KF, Byrd-Holt DD, Eberhardt MS, Flegal KM, Engelgau MM, Saydah SH, Williams DE, Geiss LS, Gregg EW: Prevalence of diabetes and impaired fasting glucose in adults in the U.S. population: national health and nutrition examination survey 1999-2002. Diabetes Care 2006, 29:1263-1268 
7. Hu DY, Pan CY, Yu JM, China Heart Survey G: The relationship between coronary artery disease and abnormal glucose regulation in China: the china heart survey. Eur Heart J 2006, 27:2573-2579.

8. The DECODE study group on behalf of the Europe an Diabetes Epidemiology Group: Glucose tolerance and mortality: comparison of WHO and american diabetes association diagnostic criteria. The DECODE study group. European diabetes epidemiology group. Diabetes epidemiology: collaborative analysis of diagnostic criteria in Europe. Lancet 1999, 354:617-621.

9. Glucose tolerance and cardiovascular mortality: comparison of fasting and 2-hour diagnostic criteria. Arch Intern Med 2001, 161:397-405.

10. Cavalot F, Petrelli A, Traversa M, Bonomo K, Fiora E, Conti M, Anfossi G, Costa G, Trovati M: Postprandial blood glucose is a stronger predictor of cardiovascular events than fasting blood glucose in type 2 diabetes mellitus, particularly in women: lessons from the San Luigi Gonzaga diabetes study. J Clin Endocrinol Metab 2006, 91:813-819.

11. Hanefeld M, Fischer S, Julius U, Schulze J, Schwanebeck U, Schmechel H, Ziegelasch HJ, Lindner J: Risk factors for myocardial infarction and death in newly detected NIDDM: the diabetes intervention study, 11-year follow-up. Diabetologia 1996, 39:1577-1583.

12. Groeneveld Y, Petri H, Hermans J, Springer MP: Relationship between blood glucose level and mortality in type 2 diabetes mellitus: a systematic review. Diab Med 1999, 16:2-13.

13. Hu Y, Liu W, Huang $R$, Zhang $X$ : Postchallenge plasma glucose excursions, carotid intima-media thickness, and risk factors for atherosclerosis in Chinese population with type 2 diabetes. Atherosclerosis 2010, 210:302-306.

14. Ceriello A, Davidson J, Hanefeld M, Leiter L, Monnier L, Owens D, Tajima N, Tuomilehto J: Postprandial hyperglycaemia and cardiovascular complications of diabetes: an update. Nutr Metab Cardiovasc Dis 2006, 16:453-456.

15. Goldberg IJ: Clinical review 124: Diabetic dyslipidemia: causes and consequences. J Clin Endocrinol Metab 2001, 86:965-971.

16. Giacco F, Brownlee M: Oxidative stress and diabetic complications. Circ Res 2010, 107:1058-1070.

17. Brownlee M: Biochemistry and molecular cell biology of diabetic complications. Nature 2001, 414:813-820

18. Stalenhoef AF, de Graaf J: Association of fasting and nonfasting serum triglycerides with cardiovascular disease and the role of remnant-like lipoproteins and small dense LDL. Curr Opin Lipidol 2008, 19:355-361.

19. Austin MA, Breslow JL, Hennekens CH, Buring JE, Willett WC, Krauss RM: Low-density lipoprotein subclass patterns and risk of myocardial infarction. JAMA 1988, 260:1917-1921.

20. Yamashina A, Tomiyama H, Takeda K, Tsuda H, Arai T, Hirose K, Koji Y, Hori S, Yamamoto Y: Validity, reproducibility, and clinical significance of noninvasive brachial-ankle pulse wave velocity measurement. Hypertens Res 2002, 25:359-364.

21. Yamashina A, Tomiyama H, Arai T, Hirose K, Koji Y, Hirayama Y, Yamamoto Y, Hori S: Brachial-ankle pulse wave velocity as a marker of atherosclerotic vascular damage and cardiovascular risk. Hypertens Res 2003, 26:615-622.

22. Farrar DJ, Green HD, Bond MG, Wagner WD, Gobbee RA: Aortic pulse wave velocity, elasticity, and composition in a nonhuman primate model of atherosclerosis. Circ Res 1978, 43:52-62.

23. van Popele NM, Grobbee DE, Bots ML, Asmar R, Topouchian J, Reneman RS, Hoeks AP, van der Kuip DA, Hofman A, Witteman JC: Association between arterial stiffness and atherosclerosis: the Rotterdam study. Stroke 2001, 32:454-460.

24. Huang $C L$, Chen MF, Jeng JS, Lin LY, Wang WL, Feng MH, Liau CS, Hwang BS, Lee YT, Su TC: Postchallenge hyperglycaemic spike associate with arterial stiffness. Int J Clin Pract 2007, 61:397-402.

25. Monnier L, Mas E, Ginet C, Michel F, Villon L, Cristol JP, Colette C: Activation of oxidative stress by acute glucose fluctuations compared with sustained chronic hyperglycemia in patients with type 2 diabetes. JAMA 2006 295:1681-1687.

26. Webb DR, Khunti K, Silverman R, Gray $\sqcup$, Srinivasan B, Lacy PS, Williams B, Davies MJ: Impact of metabolic indices on central artery stiffness: independent association of insulin resistance and glucose with aortic pulse wave velocity. Diabetologia 2010, 53:1190-1198.

27. Heart Protection Study Collaborative G, Bulbulia R, Bowman L, Wallendszus K, Parish S, Armitage J, Peto R, Collins R: Effects on 11-year mortality and morbidity of lowering LDL cholesterol with simvastatin for about 5 years in 20,536 high-risk individuals: a randomised controlled trial. Lancet 2011, 378:2013-2020.
28. Temelkova-Kurktschiev TS, Koehler C, Henkel E, Leonhardt W, Fuecker K, Hanefeld M: Postchallenge plasma glucose and glycemic spikes are more strongly associated with atherosclerosis than fasting glucose or $\mathrm{HbA} 1 \mathrm{C}$ level. Diabetes Care 2000, 23:1830-1834.

29. Maeda S, Nakanishi S, Yoneda M, Awaya T, Yamane K, Hirano T, Kohno N: Associations between small dense LDL, HDL subfractions (HDL2, HDL3) and risk of atherosclerosis in Japanese-Americans. J Arterioscler Thromb 2012, 19:444-452.

30. Berneis K, Jeanneret C, Muser J, Felix B, Miserez AR: Low-density lipoprotein size and subclasses are markers of clinically apparent and non-apparent atherosclerosis in type 2 diabetes. Metabolism 2005, 54:227-234.

31. Shoji T, Hatsuda S, Tsuchikura S, Shinohara K, Kimoto E, Koyama H, Emoto M, Nishizawa Y: Small dense low-density lipoprotein cholesterol concentration and carotid atherosclerosis. Atherosclerosis 2009, 202:582-588.

32. King GL, Shiba T, Oliver J, Inoguchi T, Bursell SE: Cellular and molecular abnormalities in the vascular endothelium of diabetes mellitus. Ann Rev Med 1994, 45:179-188.

33. Wang JJ, Yuan SY, Zhu LX, Fu HJ, Li HB, Hu G, Tuomilehto J: Effects of impaired fasting glucose and impaired glucose tolerance on predicting incident type 2 diabetes in a Chinese population with high post-prandial glucose. Diabetes Res Clin Pract 2004, 66:183-191.

34. Lawes CM, Parag V, Bennett DA, Suh I, Lam TH, Whitlock G, Barzi F, Woodward M: Blood glucose and risk of cardiovascular disease in the Asia Pacific region. Diabetes Care 2004, 27:2836-2842.

35. Yamagishi S, Nakamura K, Takeuchi M: Inhibition of postprandial hyperglycemia by acarbose is a promising therapeutic strategy for the treatment of patients with the metabolic syndrome. Med Hypotheses 2005, 65:152-154.

36. Gerber PA, Thalhammer C, Schmied C, Spring S, Amann-Vesti B, Spinas GA, Berneis K: Small, dense LDL particles predict changes in intima media thickness and insulin resistance in men with type 2 diabetes and prediabetes-a prospective cohort study. PLOS One 2013, 8:e72763.

37. Cheng Y, Du CL, Hwang JJ, Chen IS, Chen MF, Su TC: Working hours, sleep duration and the risk of acute coronary heart disease: a case-control study of middle-aged men in Taiwan. Int J Cardiol 2014, 171:419-422.

38. Ito Y, Fujimura M, Ohta M, Hirano T: Development of a homogeneous assay for measurement of small dense LDL cholesterol. Clin Chem 2011, 57:57-65.

39. Hirano $\mathrm{T}$, Ito $\mathrm{Y}$, Yoshino G: Measurement of small dense low-density lipoprotein particles. J Arterioscler Thromb 2005, 12:67-72.

doi:10.1186/1476-511X-13-179

Cite this article as: Ding et al:: Additive effects of postchallenge hyperglycemia and low-density lipoprotein particles on the risk of arterial stiffness in healthy adults. Lipids in Health and Disease 2014 13:179.

\section{Submit your next manuscript to BioMed Central and take full advantage of:}

- Convenient online submission

- Thorough peer review

- No space constraints or color figure charges

- Immediate publication on acceptance

- Inclusion in PubMed, CAS, Scopus and Google Scholar

- Research which is freely available for redistribution 